\title{
Mobility for tomorrow - with or without the internal combustion engine?
}

Prof. Dr. Peter Gutzmer, Schaeffl er AG

Die Unterlagen wurden nicht zur Veröffentlichung freigegeben.

Wir bitten um Verständnis. 